\title{
BAL QSOs AND EXTREME UFOs: THE EDDINGTON CONNECTION
}

\author{
Kastytis Zubovas ${ }^{1,2}$ and ANDREw King ${ }^{1}$ \\ ${ }^{1}$ Theoretical Astrophysics Group, University of Leicester, Leicester LE1 7RH, UK \\ 2 Center for Physical Sciences and Technology, Savanorių 231, Vilnius LT-02300, Lithuania; kastytis.zubovas@ftmc.1t \\ Received 2012 December 3; accepted 2013 April 3; published 2013 May 3
}

\begin{abstract}
We suggest a common physical origin connecting the fast, highly ionized winds (UFOs) seen in nearby active galactic nuclei (AGNs), and the slower and less ionized winds of broad absorption line (BAL) QSOs. The primary difference is the mass-loss rate in the wind, which is ultimately determined by the rate at which mass is fed toward the central supermassive black hole (SMBH) on large scales. This is below the Eddington accretion rate in most UFOs, and slightly super-Eddington in extreme UFOs such as PG1211+143, but ranges up to $\sim 10-50$ times this in BAL QSOs. For UFOs this implies black hole accretion rates and wind mass-loss rates which are at most comparable to Eddington, giving fast, highly ionized winds. In contrast, BAL QSO black holes have mildly super-Eddington accretion rates, and drive winds whose mass-loss rates are significantly super-Eddington, and so are slower and less ionized. This picture correctly predicts the velocities and ionization states of the observed winds, including the recently discovered one in SDSS J1106+1939. We suggest that luminous AGNs may evolve through a sequence from BAL QSO through LoBAL to UFO-producing Seyfert or quasar as their Eddington factors drop during the decay of a bright accretion event. LoBALs correspond to a short-lived stage in which the AGN radiation pressure largely evacuates the ionization cone, but before the large-scale accretion rate has dropped to the Eddington value. We show that sub-Eddington wind rates would produce an $M-\sigma$ relation lying above that observed. We conclude that significant SMBH mass growth must occur in super-Eddington phases, either as BAL QSOs, extreme UFOs, or obscured from direct observation.
\end{abstract}

Key words: accretion, accretion disks - black hole physics - galaxies: evolution - quasars: general

Online-only material: color figures

\section{INTRODUCTION}

Since their discovery more than a decade ago, interest in the scaling relations between supermassive black holes (SMBHs) and their galactic hosts has grown sharply. A promising candidate for the agency linking the growth of the hole with the evolution of the host is a powerful quasi-spherical wind from the central regions of an active galactic nucleus (AGN). Examples of these are widely observed. The fastest (sometimes called UFOs) are seen in a large fraction of local AGNs (Tombesi et al. 2010a, 2010b). They are detected via blueshifted X-ray absorption lines (typically helium-like iron) with velocities $\sim 0.03 c-0.15 c$ and very high ionization parameters $\xi \gtrsim 10^{4}$ (Pounds et al. 2003a, 2003b; Tombesi et al. 2010a, 2010b; Chartas et al. 2002, 2003). Broad absorption line (BAL) QSOs (Hazard et al. 1984; Knigge et al. 2008; Gibson et al. 2009) form a family with lower velocities and lower ionization. They comprise $\sim 10 \%-20 \%$ of all QSOs (Hazard et al. 1984; Knigge et al. 2008; Gibson et al. 2009) and exhibit broad blueshifted absorption lines, with low-velocity edge $v_{\min } \simeq 0.007 c-0.03 c$ and high-velocity edge $v_{\max } \simeq 0.01 c-0.06 c$ (Gibson et al. 2009). The typical ionization states in BAL QSO spectra are $\mathrm{C}$ IV, O vI, $\mathrm{N} \mathrm{v}$, with a small fraction, called LoBALs, having low-ionization lines of Mg II and Fe II.

It is clear that at least some observed winds have the properties needed to make the host galaxy sensitive to the growth of its central black hole. The quasar PG1211+143 is a UFO whose observed velocity and ionization parameter imply a momentum rate (thrust)

$$
\dot{M}_{\mathrm{w}} v_{\mathrm{w}} \simeq \frac{L_{\mathrm{Edd}}}{c},
$$

where $\dot{M}_{\mathrm{w}}$ and $v_{\mathrm{w}}$ are the mass outflow rate and velocity of the wind, and $L_{\text {Edd }}$ is the Eddington luminosity (Pounds et al. 2003a; King \& Pounds 2003). This relation suggests that the wind here is driven by photons of the AGN radiation field, Thomson-scattering once before escaping the system (King \& Pounds 2003; King 2010b). A wind with this property must have a major effect on the host galaxy, as it inevitably shocks against its interstellar gas.

This offers an obvious way for the huge binding energy of an SMBH to affect its host, and so lead to an explanation of the $M-\sigma$ relation. For black hole masses $M$ below a critical value (King 2003, 2005)

$$
M_{\sigma}=\frac{f_{g} \kappa}{\pi G^{2}} \sigma^{4} \simeq 3.7 \times 10^{8} \sigma_{200}^{4} M_{\odot}
$$

(where $f_{g}$ is the gas fraction, $\kappa$ the electron scattering opacity, and $\sigma$ the velocity dispersion of the host spheroid) the Eddington thrust of the black hole wind is too weak to lift the host interstellar gas far from the hole. The wind shock is efficiently Compton-cooled by the black hole's radiation field and falls back after sweeping up only a small mass of interstellar gas. In contrast, once the hole grows to a mass $M>M_{\sigma}$ the Eddington thrust drives this shock far enough from the hole that it no longer cools, expanding adiabatically instead. This powerful energy injection efficiently sweeps up and expels most of the host's interstellar gas. This is the probable cause (Zubovas \& King 2012a) of the observed high-speed galaxy-wide molecular outflows (Feruglio et al. 2010; Rupke \& Veilleux 2011; Sturm et al. 2011) which clear galaxy spheroids of gas and make them red and dead.

In this paper we quantitatively explore the possible range of velocities and ionization equilibria in AGN winds. Our 
results suggest that BAL QSO winds and extreme UFOs like PG1211+143 are manifestations of the same physical process. The primary difference between the two classes is the mass outflow rate in the wind, which has its ultimate origin in the mass inflow rate toward the accreting SMBH from large scales. In BAL QSOs, this large-scale inflow rate is highly superEddington, leading to a dense and slow wind outflow, while extreme UFOs have more moderate inflow rates $\dot{M}_{\text {acc }} \sim \dot{M}_{\text {Edd }}$. Variations in the geometry of the accretion flow can account for the large spread in outflow velocities for the same ionization species or the same object, and vice versa. All these winds transmit momentum rates $\gtrsim L_{\text {Edd }} / c$ to the host interstellar medium (ISM), and so would correctly predict the observed $M-\sigma$ relation.

In contrast, the majority of UFOs have mass inflow rates which are significantly sub-Eddington. If these rates prevailed throughout the growth of the SMBH they would produce either an $M-\sigma$ relation lying significantly above what is seen, or no relation at all. We conclude that significant SMBH mass growth occurs in (super) Eddington phases. These manifest themselves either as BAL QSOs or extreme UFOs, or correspond to obscured systems.

This paper is structured as follows. We review the properties of AGN winds in Section 2. We then describe the model for calculating the relation between wind ionization and velocity, and present the results (Section 3). Finally, we discuss the physical interpretation and implications of our findings in Section 4.

\section{AGN WINDS}

The launching of winds from accreting AGN is discussed in King (2010b). Here we present a brief overview, concentrating on the properties relevant for the connection between UFOs and BAL QSOs: wind ionization, velocity, and mass outflow rate. We first consider the likely range of accretion rates.

\subsection{Accretion Rates in AGN}

In an accretion flow around an $\mathrm{SMBH}$, the accretion rate at each radius is determined by local properties and the ultimate process feeding it, rather than directly by the black hole itself. There is no reason that this rate should respect the Eddington limit for the black hole - at radii larger than the point of direct infall to the hole, the Eddington limit may be significantly exceeded. The properties of the host galaxy set a limit on the infall rate $\dot{M}_{\text {in }}$ onto the black hole accretion disk at large radii. This cannot be larger than the rate given by allowing gas previously in equilibrium suddenly to fall freely: for a roughly isothermal equilibrium with velocity dispersion $\sigma \equiv 200 \sigma_{200} \mathrm{~km} \mathrm{~s}^{-1}$ this dynamical rate is

$$
\dot{M}_{\text {dyn }} \sim \frac{f_{\mathrm{g}} \sigma^{3}}{G} \simeq 2 \times 10^{3} \sigma_{200}^{3} M_{\odot} \mathrm{yr}^{-1}
$$

where $f_{\mathrm{g}}$ is the gas fraction, i.e., the ratio of gas density in the galaxy to the total density; $f_{\mathrm{g}} \simeq 0.16$ is the cosmological value. Since $\dot{M}_{\text {in }}<\dot{M}_{\text {dyn }}$ this shows that

$$
\frac{\dot{M}_{\text {in }}}{\dot{M}_{\text {Edd }}}<40 \frac{M_{\sigma}}{M \sigma_{200}},
$$

where $\dot{M}_{\text {Edd }} \simeq 2 M_{8} M_{\odot} \mathrm{yr}^{-1}$ is the Eddington accretion rate (with $M_{8}=M / 10^{8} M_{\odot}$ and accretion efficiency $\eta \simeq 0.1$ ),
$M_{\sigma} \simeq 3.67 \times 10^{8} \sigma_{200}^{4} M_{\odot}$ is the critical black hole mass (King 2010b; Zubovas \& King 2012b), and $M$ is the current black hole mass. Clearly, unless the black hole mass is significantly below $M_{\sigma}$, or the galaxy has $\sigma \ll 200 \mathrm{~km} \mathrm{~s}^{-1}$, the maximum mass inflow rate feeding the accretion disk is $\lesssim 50$ times the Eddington limit. Since the dynamical rate is an extreme upper limit this suggests that AGN are never fed at very high Eddington rates.

\subsection{Wind Launching in Super-Eddington Systems}

An accretion disk reacts to a locally super-Eddington inflow by driving away the excess as a wind at each radius. Shakura \& Sunyaev (1973) show that this mass loss implies that the total luminosity of the system is

$$
L=L_{\text {Edd }}(1+\ln (1+\dot{m})),
$$

where

$$
\dot{m} \equiv \frac{\dot{M}_{\mathrm{w}}}{\dot{M}_{\mathrm{acc}}}=\frac{\dot{M}_{\mathrm{in}}}{\dot{M}_{\mathrm{acc}}}-1,
$$

with $\dot{M}_{\text {w }}$ the wind outflow rate and $\dot{M}_{\text {acc }}$ the black hole accretion rate.

We have seen above that the accretion rate and the wind are never very super-Eddington, so the wind optical depth to electron scattering is $\sim 1$. This means that each photon emitted by the accretion onto the black hole scatters about once before escaping. As electron scattering is front-back symmetric, this shows that the wind acquires a momentum flow rate

$$
\dot{M}_{\mathrm{w}} v_{\mathrm{w}} \simeq \frac{L}{c},
$$

(King 2010b) where $L$ is the total black hole and disk luminosity (Equation (5)).

\subsection{Wind Launching in Sub-Eddington Systems}

In a sub-Eddington AGN, Compton scattering is by definition incapable of launching winds. However, radiation may still be able to expel matter in a wind by line-driving (see below): hot stars achieve this, for example. For disk accretion this process is formally identical to the super-Eddington case considered in the previous subsection, except that the Eddington luminosity is replaced by a lower luminosity $L_{\text {crit }}$. The analogous equation to Equation (5) holds, i.e.,

$$
L=L_{\text {crit }}(1+\ln (1+\dot{m})),
$$

where again

$$
\dot{m} \equiv \frac{\dot{M}_{\mathrm{w}}}{\dot{M}_{\mathrm{acc}}}=\frac{\dot{M}_{\mathrm{in}}}{\dot{M}_{\mathrm{acc}}}-1 .
$$

The analogy with the super-Eddington case goes deeper. Linedriving, i.e., scattering off bound electrons, has to accelerate the wind to the escape velocity from the photosphere in the immediate vicinity of the accreting black hole. Thus, absorption lines with rest energies lying below the peak of the continuum spectrum (largely a black body at the effective temperature of about $10^{5} \mathrm{~K}$ ) are blueshifted across most of the spectrum and so absorb and re-radiate (i.e., scatter) almost all of the bolometric luminosity. In a similar manner to Equation (7) this gives

$$
\dot{M}_{\mathrm{w}} v_{\mathrm{w}} \simeq \frac{L_{\mathrm{bol}}}{c},
$$

which is essentially the argument of Cassinelli \& Castor (1973). Figure 4 of Tombesi et al. (2013) suggests that this approximate relation may hold for a significant number of UFOs. 

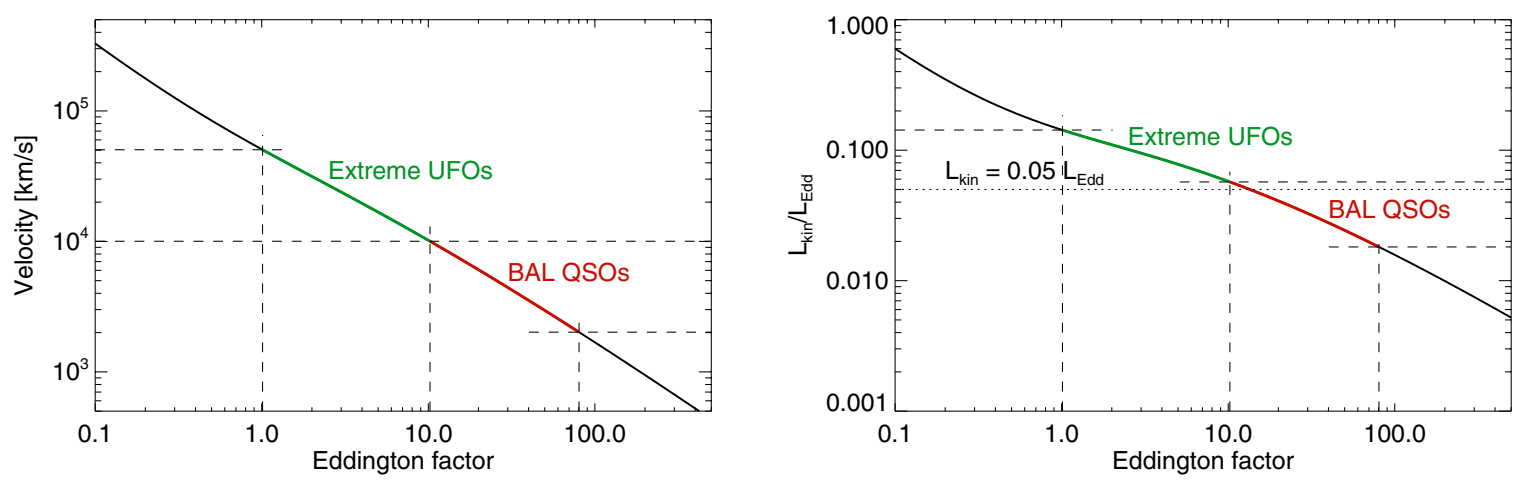

Figure 1. Left: wind velocity as function of the Eddington factor, from Equation (11). Horizontal dashed lines show approximate limits of observed velocities of UFOs $\left(10^{4}-5 \times 10^{4} \mathrm{~km} \mathrm{~s}^{-1}\right)$ and BAL QSO outflows $\left(2000-10000 \mathrm{~km} \mathrm{~s}^{-1}\right)$, with corresponding $\dot{m}$ factors identified. Right panel: wind kinetic luminosity as function of the Eddington factor, from Equation (12).

(A color version of this figure is available in the online journal.)

\subsection{Wind Properties}

The wind velocity $v_{w}$ in both sub- and super-Eddington systems can be expressed in terms of $\dot{m}$ :

$$
v_{\mathrm{w}}=\frac{\eta \dot{M}_{\mathrm{acc}} c^{2}[1+\ln (1+\dot{m})]}{\dot{M}_{\mathrm{w}} c}=\eta c \frac{1+\ln (1+\dot{m})}{\dot{m}} .
$$

The wind kinetic luminosity is

$$
L_{\mathrm{kin}}=\frac{1}{2} \dot{M}_{\mathrm{w}} v_{\mathrm{w}}^{2}=\frac{\eta}{2} L \frac{[1+\ln (1+\dot{m})]^{2}}{\dot{m}} .
$$

We plot these two relations in Figure 1 for $\eta=0.1$ and $L=L_{\text {Edd }}$, declining below this value for UFOs. We identify the typical ranges of UFO and BAL QSO outflow velocities (green and red, respectively) together with the corresponding $\dot{m}$ factors and kinetic luminosities; we note, however, that there is an overlap in velocity between the two populations, as described in the Introduction.

The gas density in the outflowing wind follows from the mass outflow rate at radius $R$ :

$$
n_{\mathrm{w}}=\frac{\dot{M}_{\mathrm{w}}}{4 \pi R^{2} m_{\mathrm{p}} v_{\mathrm{w}}},
$$

with $m_{\mathrm{p}}$ the proton mass. UFOs are generally believed to have large covering factors (i.e., $\Omega_{\mathrm{w}} / 2 \pi \gtrsim 0.6$; Tombesi et al. 2010a, $2010 b)$ so we do not consider potential outflow collimation. This gives the wind ionization parameter far from the hole as

$\xi_{\mathrm{w}}=\frac{L_{\mathrm{ion}}}{n_{\mathrm{w}} R^{2}}=\frac{b l_{\mathrm{i}} L \times 4 \pi m_{\mathrm{p}} v_{\mathrm{w}}}{\dot{M}_{\mathrm{w}}} \simeq 4 \pi m_{\mathrm{p}} \eta^{2} c^{3} \frac{1+\ln (1+\dot{m})}{\dot{m}^{2}} b l_{\mathrm{i}}$,

where $l_{i}$ is the fraction of the AGN luminosity capable of ionizing a particular species and $b \leqslant 1$ is a quasar radiation beaming factor. (Strongly super-Eddington inflow may lead to significant beaming (e.g., King 2009), and we consider its effects in the Discussion.) For the moment we keep $b$ as a free parameter. We assume that the disk does not contribute to ionization; this is reasonable considering that the disk radiates in the UV and longer wavelengths. Had we assumed the disk spectrum to be the same as the SMBH spectrum, an extra factor $1+\ln (1+\dot{m})$ would have appeared in the expression for $\xi$.
Evaluating the constants in Equation (14) gives

$$
\xi_{\mathrm{w}} \simeq 5.7 \times 10^{4} \eta_{0.1}^{2} \frac{1+\ln (1+\dot{m})}{\dot{m}^{2}} b \frac{l_{\mathrm{i}}}{10^{-2}},
$$

where we have parameterized $\eta_{0.1} \equiv \eta / 0.1$. Crucially, none of the wind parameters depend on the Eddington ratio of the AGN directly, but only through $\dot{m}$. The high ionization parameter predicted for $\dot{m} \sim 1$ explains why UFO winds are generally detected through Fe XXV and Fe XXVI absorption lines (cf. King 2010a).

Equation (15) implies a set of self-consistent solutions for $\xi, l_{\mathrm{i}}$ specified by $\dot{m}, b$, and $\eta$. The ionization parameter $\xi_{\mathrm{w}}$ depends linearly on the ionizing fraction of the AGN luminosity. However, species of higher ionization level (corresponding to higher ionization parameter) have higher threshold energies for ionization, leading to a lower ionizing fraction $l_{\mathrm{i}}$. Hence, for a given set of parameters $b, \eta$, and $\dot{m}$, there is at most one possible solution where the ionization parameter calculated from Equation (14) corresponds to the ionized species as specified by $l_{\mathrm{i}}$. Conversely, different combinations of these parameters can lead to different solutions of the ionization equilibrium. In the next section, we estimate the ionization equilibria for seven elements corresponding to various parameter ranges. We will show that our model predicts the ionized species observed in BAL QSOs to move with velocities consistent with observations.

\section{MODEL AND RESULTS}

\subsection{Numerical Model}

To find the connection between gas inflow parameters and self-consistent wind ionization solutions, we use a simple numerical approach. We consider seven elements commonly observed in BAL QSO spectra-carbon, nitrogen, oxygen, magnesium, silicon, sulfur, and iron. Their ionized species cover a large range of BAL QSO parameters.

We start by constructing a "typical" quasar spectral energy distribution (SED), using data from Elvis et al. (1994) and Winter et al. (2012). The SED is composed of five components: a rising radio and sub-mm continuum, flat IR, optical, and UV background with thermal IR and UV bumps superimposed, and a cutoff power-law at X-ray and higher energies. We varied the SED to account for the observational uncertainties and found that the overall results remain unchanged. Using the SED, we 


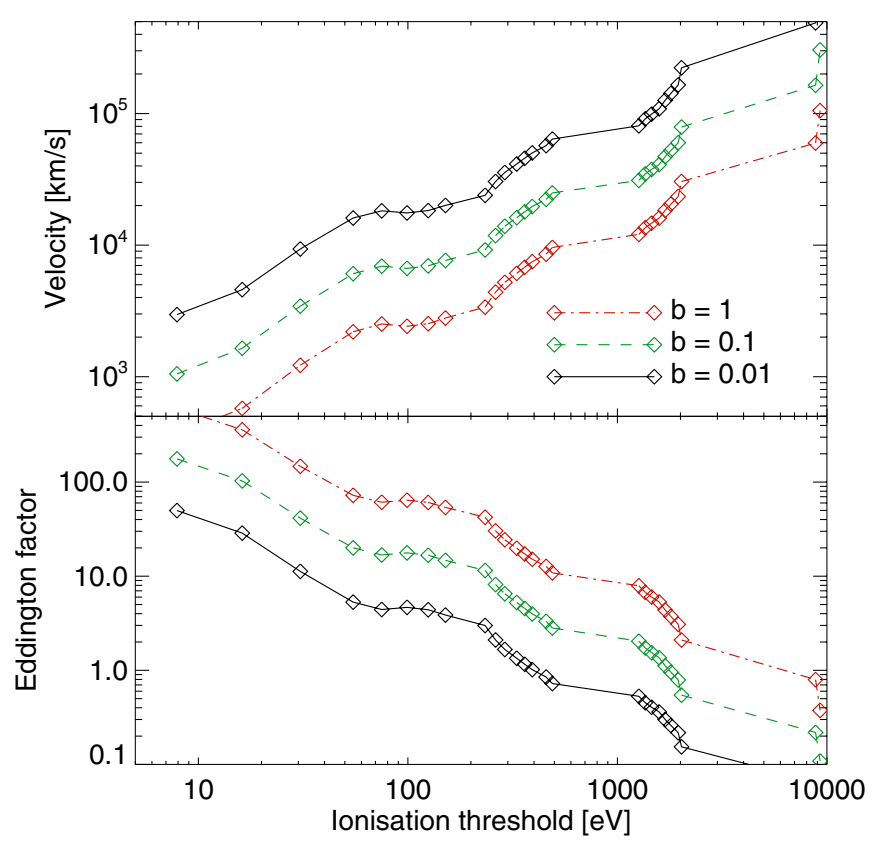

Figure 2. Wind velocity (top) and Eddington factor (bottom) as function of iron ionization level (diamonds) for three values of the luminosity beaming factor: $b=0.01$ (black solid curve), 0.1 (green dashed), and 1 (red dot-dashed).

(A color version of this figure is available in the online journal.)

find the fraction of total luminosity that can ionize a particular element to any given level:

$$
l_{i, j}=\int_{E_{i, j}}^{\infty} E_{\mathrm{SED}} d E,
$$

where $E_{\mathrm{SED}}$ is the energy at a given energy and integration is carried out above the ionization threshold of level $i$ for element $j$. The SED is normalized by construction.

Next, we consider the fractional abundances of various elemental ionization levels in astrophysical plasmas as function of temperature. We use data from Jordan (1969) for all elements except iron; for the latter, we take the data from Arnaud \& Raymond (1992), since Jordan (1969) does not give a complete ionization table for the element. We convert each temperature to a corresponding ionization parameter using a prescription based on the work of Sazonov et al. (2005). The prescription is valid for an optically thin plasma illuminated by, and in thermal equilibrium with, a quasar radiation field; both assumptions are satisfied when considering equilibrium ionization structures in a diffuse wind. In the few cases where temperatures fall outside the range considered by Sazonov et al. (2005), we adopt a simplified relation $\xi=T / 200$ (in cgs units), which follows the approximate analytical relation (Sazonov et al. 2004). Given the fractional abundances and the corresponding ionization parameters, we calculate the weighted average ionization parameter for each ionization level of each element. This is the $\xi_{\mathrm{w}}$ required for equilibrium.

We then substitute the calculated values of $\xi_{\mathrm{w}}$ and $l_{i, j}$ into Equation (15) and isolate the quantity

$$
\frac{1+\ln (1+\dot{m})}{\dot{m}^{2}}=\frac{\xi_{\mathrm{w}}}{5.7 \times 10^{6} l_{i, j} b \eta_{0.1}^{2}}
$$

for each element. We now use Equations (11) and (17) to find $v_{w}$ and $\dot{m}$ numerically for $b=0.01,0.1,1$, assuming $\eta=0.1$.

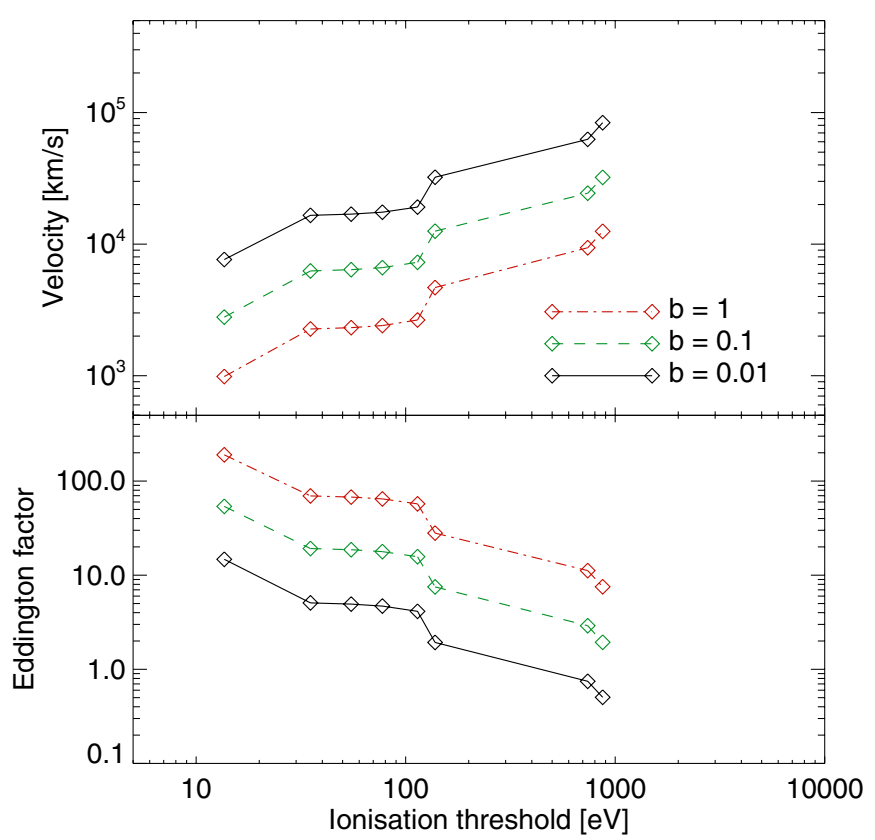

Figure 3. Same as Figure 2, but for oxygen.

(A color version of this figure is available in the online journal.)

\subsection{Extreme UFO Solution}

Figure 2 shows the outflow velocity and Eddington factor given by Equations (11) and (17) for all iron species. Each diamond corresponds to a different ionization level, increasing from Fe II to Fe Xxvi. The three curves are for $b=0.01$ (black solid), $b=0.1$ (green dashed), and $b=1$ (red dot-dashed).

The two rightmost points in the figure are for helium- and hydrogen-like iron ions, and describe mildly relativistic extreme UFO winds. For the expected unbeamed luminosities $(b=1)$ the outflow has $\dot{m} \lesssim 1$, i.e., the accretion rate is not much higher than Eddington. The predicted outflow velocities are $\sim 0.15 c-0.3 c$, within the observed range (Tombesi et al. 2010a, 2010b).

\subsection{BAL QSO Solution}

In Figure 3, we plot the same data as in Figure 2, but for oxygen ionization states. BAL QSOs are often observed via an O VI absorption line (e.g., Baldwin et al. 1996), so we concentrate on the results for this ion (fifth diamond from the left). It is clear that the major difference from Seyfert winds is the significantly higher Eddington factor $\dot{m} \sim 10$. For flows with spherically symmetric radiation fields $(b=1)$, this ionization state is consistent with $v_{\mathrm{w}, \mathrm{O}} \simeq 2500 \mathrm{~km} \mathrm{~s}^{-1}$, close to the lower bound of the minimum BAL QSO outflow velocities (Gibson et al. 2009). However, if the Seyfert luminosity is beamed perpendicular to the disk plane, the velocity is higher. For a beaming factor $b=0.1$, we find $v_{\mathrm{w}, \mathrm{O}} \simeq 7000 \mathrm{~km} \mathrm{~s}^{-1}$, well within the range of observed BAL QSO parameters. Other ions-C IV, N v, Si IV, S IV, and S VI-show very similar results. We compare our results with the range of minimum, rather than the maximum, BAL QSO outflow velocities, because we only consider radiative acceleration of winds. The wind is usually launched with considerable velocity (similar to the escape velocity at the launch radius) by gas pressure, which would increase its final velocity as well.

To quantify the constraints on luminosity beaming for BAL QSO outflows, we plot $b$ for eight common ions giving wind velocities $v_{\mathrm{w}}=2000-10^{4} \mathrm{~km} \mathrm{~s}^{-1}$ (Figure 4). The six ions most 


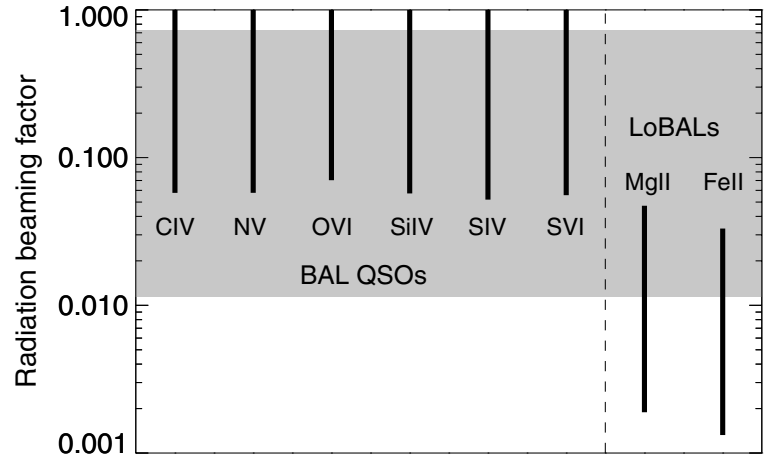

Figure 4. Range of beaming factors giving ionization equilibrium solutions consistent with the lower end of BAL QSO wind velocities $v_{\mathrm{w}}=2000-10^{4} \mathrm{~km} \mathrm{~s}^{-1}$ for commonly observed BAL QSO ions. Higher beaming factors correspond to lower velocities. Low ionization species $\mathrm{Mg}$ II and Fe II are seen in a small fraction of BAL QSOs, suggesting a short-lived evolutionary phase. The shaded region shows beaming factors corresponding to the accretion rates $\dot{m}$ between 10 and 50, based on King (2009).

commonly observed in BAL QSOs-C IV, N v, O VI, Si IV, S IV, and $\mathrm{S}$ vI-all have solutions with $0.08 \lesssim b \lesssim 1$. The shaded region corresponds to the range of beaming factors predicted by Equation (8) of King (2009) for $\dot{m}$ values between 10 and 80.

\subsection{LoBAL Solution}

The presence of two more ions-Mg II and Fe II-in this simple picture is consistent with the observed outflow velocities only if the AGN radiation field is strongly beamed, with $b \simeq 0.01$. Such strong beaming may not be required if other processes can reduce the ionization level in these systems, but our simple model does not take such effects into account. We comment further on the validity of this model and several possible complicating issues in the next section.

\section{DISCUSSION}

The results of the last section suggest that the main parameter affecting the properties of an outflowing radiation-pressure driven wind in AGN is its Eddington factor $\dot{m}$, and so ultimately the inflow rate from the host galaxy toward the central SMBH. This is $\sim 1$ in extreme UFO-producing AGN, but $\gtrsim 10$ in BAL QSOs. We can draw several conclusions from this.

\subsection{BAL QSO Fraction}

BAL QSOs comprise a small fraction, 15\%, of all AGNs (Knigge et al. 2008). In our picture this follows from the requirement for inflow from the host galaxy at significantly super-Eddington rates. From Equation (4) these conditions are more easily achieved in galaxies with low values of $\sigma$ or SMBHs with masses well below the $M-\sigma$ relation, but both cases correspond to low absolute luminosities and so are disfavored by observation. So the rarity of BAL QSOs suggests that neardynamical inflow rates in big galaxies with big black holes are rare, presumably because such episodes are short lived or obscured.

\subsection{Absorption Line Widths}

The fact that the accretion disk contributes significantly to driving the wind may explain the width of BAL QSO absorption lines. Since the wind is launched from a wide range of radii in the disk, its gas has a wide range of initial velocities. Even though they are all accelerated by the central source, the original spread remains.

\subsection{Warm Absorbers}

A large fraction of Seyfert galaxies, as well as some quasars, show absorption in the UV and soft X-ray spectra of material moving with comparatively low velocities, $v_{\mathrm{WA}}=10-1000 \mathrm{~km} \mathrm{~s}^{-1}$ (McKernan et al. 2007) with line widths similar to these velocities. They are composed of weakly ionized $\left(\xi \simeq 30 \mathrm{erg} \mathrm{cm} \mathrm{s}^{-1}\right.$ ) $10^{5} \mathrm{~K}$ gas (e.g., Reynolds \& Fabian 1995). Ionization models show that these "warm absorbers" exist outside the broad line regions of their AGN and are perhaps associated with the gas in the torus (Blustin et al. 2005). Comparison of these systems with typical Seyferts suggests that they should be accreting with high Eddington ratios $\dot{m}$ (Brandt et al. 2000).

The model presented in this paper does not account for multiple ionization levels in an outflow. Nevertheless, a simple qualitative picture can be developed to explain these. The wind rising from the accretion disk around an AGN is stratified in density, with slower and denser material close to the disk and faster lower density material further away. As this wind is illuminated by an isotropic AGN radiation field, its ionization parameter increases with height above the disk mid-plane. For a Gaussian vertical density profile, the particle density drops (and the ionization level increases) by a factor 1000 within less than three scale heights from the base of the wind; this difference would allow both warm absorbers and hydrogen-like iron to coexist within the same outflow. A similar model was proposed for the weak outflow in NGC 5548 (Steenbrugge et al. 2005); we claim that this is the general case.

In addition, any inhomogeneities present in the ISM surrounding the AGN can produce lower ionization features in the spectrum. Such features of varying velocities and ionization levels have been identified in both UFOs (e.g., Pounds \& Vaughan 2011) and BAL QSOs (e.g., Moe et al. 2009). Finally, it is possible that low-velocity, low-ionization species are formed by recombination in the coolest part of the post-shock flow (K. A. Pounds \& A. King 2013, in preparation).

\subsection{What are LoBALs?}

Our work suggests that AGN winds and BAL QSO outflows are explicable by the same physical mechanism, the only difference being the large-scale properties of the reservoir feeding the black hole. The LoBALs appear to be a rare state in which the AGN radiation is somehow beamed away from most of the outflow.

We speculate that AGNs may evolve over time from an initial BAL QSO state of an AGN, through a LoBAL state, to a final stage as a Seyfert galaxy or a quasar (see Figure 5 for a schematic of the process). We assume that an AGN accretion episode starts when some process creates a large reservoir of gas of relatively low angular momentum around the SMBH (left panel of the figure). This would give a near-dynamical inflow rate $\dot{M}_{\text {in }} \lesssim M_{\text {dyn }}$ and hence a highly super-Eddington SMBH accretion rate $(\dot{m} \gg 1)$. The black hole and its accretion disk begin driving an outflow, which is observed in BALs, with the accretion luminosity being somewhat beamed $(b \gtrsim 0.1)$, giving a BAL QSO.

The radiation pressure inside the beamed ionizing cone is $p_{\text {r,beam }} \propto b^{-1}$, where $b$ probably decreases with $\dot{m}$ (King 2009 suggests that $b \propto \dot{m}^{-2}$ ), reinforcing the tendency of larger accretion rates to give higher radiation pressure. The pressure inside the ionization cone is much higher than the pressure in the more modestly illuminated vicinity of 


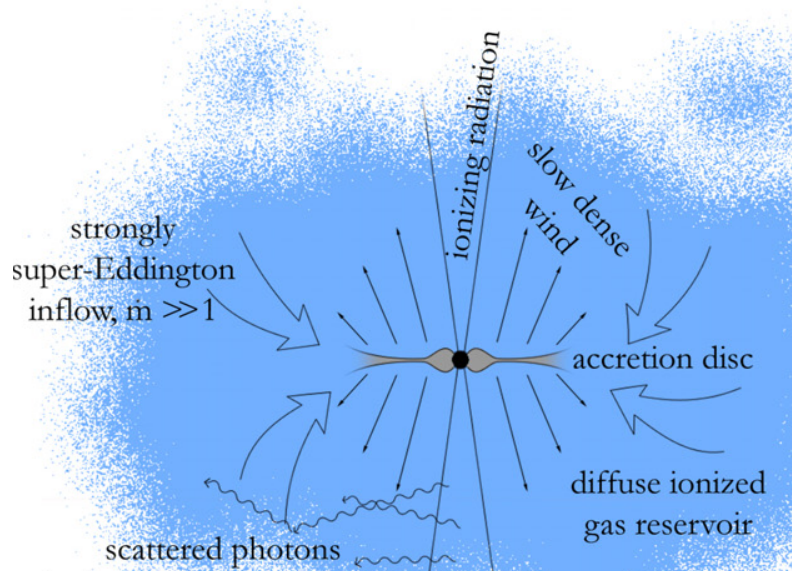

ionize surrounding material
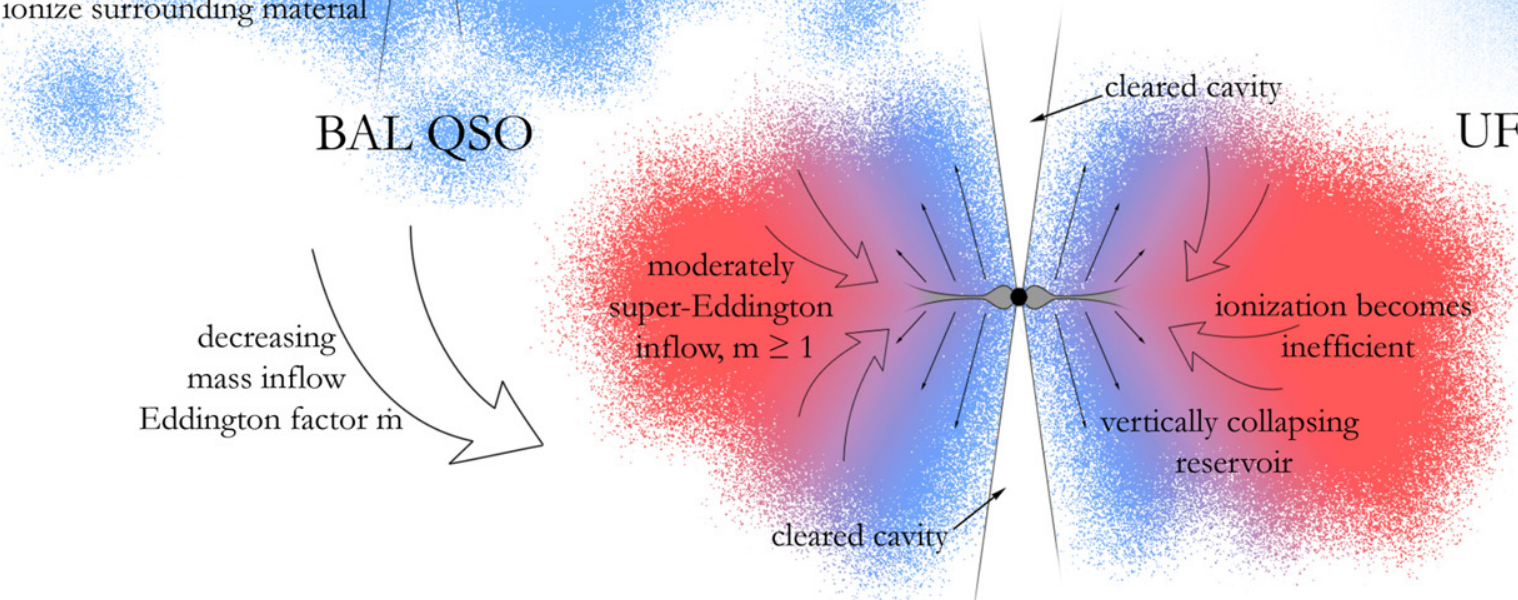

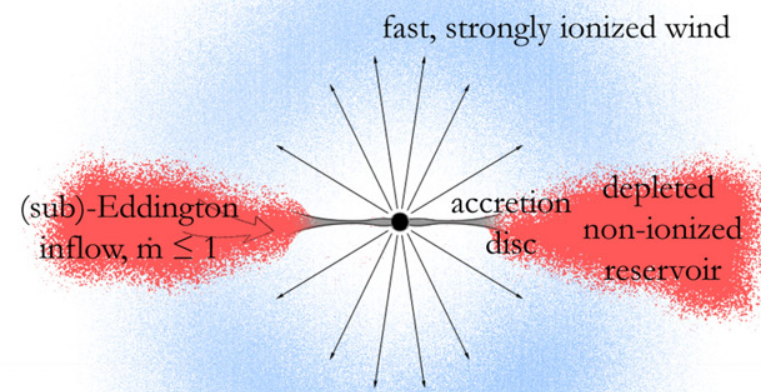

isotropic ionizing radiation

\section{UFO-producing AGN}

\section{LoBAL}

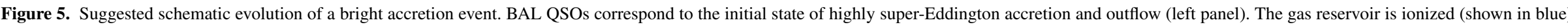

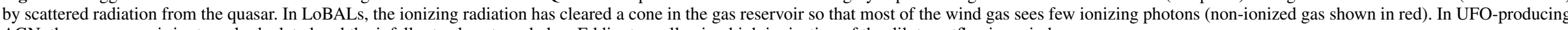
AGN, the gas reservoir is strongly depleted and the infall rate close to or below Eddington, allowing high ionization of the dilute outflowing wind.

(A color version of this figure is available in the online journal.) 
the disk, $p_{\mathrm{r}, \mathrm{d}} \propto(1+\ln (1+\dot{m}))$. As a result, the beamed ionizing radiation flux evacuates a conical cavity from the surrounding material. This process, together with the inevitable vertical collapse of the large-scale reservoir as it circularizes close to the $\mathrm{SMBH}$, reduces the effective beaming factor severely (to $b \sim 10^{-2}$ ) as there is less material interacting with the beamed radiation of the SMBH. This stage corresponds to a LoBAL (middle panel).

The large-scale reservoir feeding the AGN is depleted in a few dynamical times $t_{\mathrm{d}} \simeq 5 \times 10^{4} R_{10} \sigma_{200}^{-1} \mathrm{yr}$, with $R_{10}$ the size of the reservoir in tens of parsecs. As this happens, the Eddington factor $\dot{m}$ drops until eventually only the accretion disk remains. The SMBH then starts to accrete at $\dot{m} \simeq 1$, producing a UFOtype wind.

Both changes to the system state depend on dynamical processes (reservoir collapse and depletion), so they happen soon after one another. This may explain why LoBALs are rare-they are systems in a process of changing from BAL QSOs to more modestly accreting AGN.

\subsection{A Particular Example: SDSS J1106+1939}

A recently discovered powerful BAL QSO outflow in SDSS J1106+1939 (Borguet et al. 2013) has a mass flow rate of $400 M_{\odot} \mathrm{yr}^{-1}$ and velocity $v \simeq 8000 \mathrm{~km} \mathrm{~s}^{-1}$. From Equation (11) we find that this velocity corresponds to $\dot{m}=14$, while Equation (12) gives $L_{\text {kin }}=0.05 L_{\text {Edd }}$ for this system, exactly as observed. The Eddington ratio also implies an Eddington accretion rate of $\sim 29 M_{\odot} \mathrm{yr}^{-1}$, giving an SMBH mass $M_{\mathrm{SMBH}}=$ $1.3 \times 10^{9} M_{\odot}$, almost exactly equal to the $\sim 1.5 \times 10^{9} M_{\odot} \mathrm{SMBH}$ mass calculated from the observed kinetic luminosity.

Borguet et al. (2013) determine the scale of the flow as about $300 \mathrm{pc}$, considerably smaller than the likely Compton cooling radius $\left(\sim 3 \mathrm{kpc}\right.$ for a black hole of mass $M_{\mathrm{SMBH}}=1.3 \times 10^{9} M_{\odot}$ lying close to the $M-\sigma$ relation; see Zubovas \& King 2012b). So it is likely that the collision of the observed wind with the host gas will result in a strongly cooled shock, and not sweep the galaxy clear of gas in an energy-driven, possibly molecular, outflow.

\section{CONCLUSION}

We have shown that the outflowing winds in Seyfert galaxies and BAL QSOs differ only in their mass outflow rates. These are on the order of the Eddington accretion rate in Seyferts, but $\sim 10-50$ times this in BAL QSOs. Our picture correctly predicts the velocities and ionization states of the observed winds, including the recently discovered case of SDSS J1106+1939. We suggest that luminous AGNs may evolve from BAL QSOs through LoBAL to Seyfert as their Eddington factors drop during the decay of a bright accretion event. LoBALs correspond to a short-lived stage in which the AGN radiation pressure largely evacuates the ionization cone, but before the large-scale accretion rate has dropped to Eddington value.

We note finally that Equations (5) and (7) imply that extreme UFOs and BAL QSO winds exert the Eddington thrust on the interstellar gas of their host galaxies, and so produce the $M-\sigma$ relation (2), which agrees with observation. The analogous Equations (5) and (10) for sub-Eddington UFOs would instead produce an $M-\sigma$ relation with the black hole masses larger by factors $\simeq L_{\text {Edd }} / L_{\text {bol }}$, which can approach 100 in some cases. Since larger masses should be easier to measure than smaller ones, it seems unlikely that such a relation holds in reality. We therefore conclude that such sub-Eddington systems cannot be the sites of significant SMBH mass growth. Unless this occurs in obscurity, most galaxies must pass through prolonged phases as extreme UFOs or BAL QSOs.

We thank Mike Goad and Belinda Wilkes for enlightening discussions regarding observed features of BAL QSOs. We are particularly grateful to the anonymous referee for bringing to our attention several issues that helped us improve the paper considerably. K.Z. was supported by an STFC studentship and later by an STFC Rolling Grant for Theoretical Astrophysics.

\section{REFERENCES}

Arnaud, M., \& Raymond, J. 1992, ApJ, 398, 394

Baldwin, J. A., Ferland, G. J., Korista, K. T., et al. 1996, ApJ, 461, 664

Blustin, A. J., Page, M. J., Fuerst, S. V., Branduardi-Raymont, G., \& Ashton, C. E. 2005, A\&A, 431, 111

Borguet, B. C. J., Arav, N., Edmonds, D., Chamberlain, C., \& Benn, C. 2013, ApJ, 762, 42

Brandt, W. N., Laor, A., \& Wills, B. J. 2000, ApJ, 528, 637

Cassinelli, J. P., \& Castor, J. I. 1973, ApJ, 179, 189

Chartas, G., Brandt, W. N., \& Gallagher, S. C. 2003, ApJ, 595, 85

Chartas, G., Brandt, W. N., Gallagher, S. C., \& Garmire, G. P. 2002, ApJ, 579,169

Elvis, M., Wilkes, B. J., McDowell, J. C., et al. 1994, ApJS, 95, 1

Feruglio, C., Maiolino, R., Piconcelli, E., et al. 2010, A\&A, 518, L155

Gibson, R. R., Jiang, L., Brandt, W. N., et al. 2009, ApJ, 692, 758

Hazard, C., Morton, D. C., Terlevich, R., \& McMahon, R. 1984, ApJ, 282, 33

Jordan, C. 1969, MNRAS, 142, 501

King, A. 2003, ApJL, 596, L27

King, A. 2005, ApJL, 635, L121

King, A. R. 2009, MNRAS, 393, L41

King, A. R. 2010a, MNRAS, 408, L95

King, A. R. 2010b, MNRAS, 402, 1516

King, A. R., \& Pounds, K. A. 2003, MNRAS, 345, 657

Knigge, C., Scaringi, S., Goad, M. R., \& Cottis, C. E. 2008, MNRAS, 386, 1426

McKernan, B., Yaqoob, T., \& Reynolds, C. S. 2007, MNRAS, 379, 1359

Moe, M., Arav, N., Bautista, M. A., \& Korista, K. T. 2009, ApJ, 706, 525

Pounds, K. A., King, A. R., Page, K. L., \& O'Brien, P. T. 2003a, MNRAS, 346, 1025

Pounds, K. A., Reeves, J. N., King, A. R., et al. 2003b, MNRAS, 345, 705

Pounds, K. A., \& Vaughan, S. 2011, MNRAS, 413, 1251

Reynolds, C. S., \& Fabian, A. C. 1995, MNRAS, 273, 1167

Rupke, D. S. N., \& Veilleux, S. 2011, ApJL, 729, L27

Sazonov, S. Y., Ostriker, J. P., Ciotti, L., \& Sunyaev, R. A. 2005, MNRAS, 358,168

Sazonov, S. Y., Ostriker, J. P., \& Sunyaev, R. A. 2004, MNRAS, 347, 144

Shakura, N. I., \& Sunyaev, R. A. 1973, A\&A, 24, 337

Steenbrugge, K. C., Kaastra, J. S., Crenshaw, D. M., et al. 2005, A\&A, 434, 569

Sturm, E., González-Alfonso, E., Veilleux, S., et al. 2011, ApJL, 733, L16

Tombesi, F., Cappi, M., Reeves, J. N., et al. 2010a, A\&A, 521, A57

Tombesi, F., Cappi, M., Reeves, J. N., et al. 2013, MNRAS, 430, 1102

Tombesi, F., Sambruna, R. M., Reeves, J. N., et al. 2010b, ApJ, 719, 700

Winter, L. M., Veilleux, S., McKernan, B., \& Kallman, T. R. 2012, ApJ, 745, 107

Zubovas, K., \& King, A. 2012a, ApJL, 745, L34

Zubovas, K., \& King, A. R. 2012b, MNRAS, 426, 2751 